\title{
Near-infrared observations of the carbon stars TU Geminorum and SS Virginis at milliarcsecond resolution (Research Note)
}

\author{
A. Richichi ${ }^{1}$ and T. Chandrasekhar ${ }^{2}$ \\ ${ }^{1}$ European Southern Observatory, Karl-Schwarzschildstr. 2, 85748 Garching bei München, Germany \\ e-mail: arichich@eso.org \\ 2 Physical Research Laboratory, Navrangpura, 380009 Ahmedabad, India \\ e-mail: chandra@prl.res.in
}

Received 9 December 2005 / Accepted 5 February 2006

\begin{abstract}
Aims. High-angular resolution investigations of carbon stars.

Methods. Milliarcsecond resolution observations in the near infrared $K$ band by the method of lunar occultations are presented for the carbon stars TU Gem and SS Vir.

Results. Although a companion to TU Gem is known from the Tycho catalogue, we were not able to detect it so we place limits on its $K$-band brightness. For both stars, we determined accurate values of the angular diameters at the phase of the observation, namely $8.37 \pm 0.07$ mas and $4.62 \pm 0.15$ mas for SS Vir and TU Gem. Using distance estimates from the literature, we found that the two stars have radii of about $500 R_{\odot}$ and $250 R_{\odot}$, respectively. We also computed their effective temperatures.

Conclusions. The result for SS Vir $(2445 \pm 40 \mathrm{~K})$ is in good agreement with estimates, but in the case of TU Gem our value of $3160 \pm 110 \mathrm{~K}$ requires reconsidering currently available models.
\end{abstract}

Key words. occultations - techniques: high angular resolution - stars: fundamental parameters - stars: individual: TU Gem stars: individual: SS Vir

\section{Introduction}

Carbon stars pose a challenge to the theoretical understanding of the late evolutionary stages of stars with a moderate mass. Models of carbon stars have traditionally lacked sufficient observational data and constraints, particularly for what concerns their angular sizes and their immediate circumstellar environment. This is partly due to the rarity of the carbon star phenomenon, which makes only a few objects observationally accessible in combined terms of brightness and angular resolution. Angular diameters allow us to estimate effective temperatures, along with the linear radii if a reliable distance determination is available. Clearly, directly determining the angular diameters of carbon stars constitutes a key factor for our understanding of these class of stars, including their pulsational properties and their thermal cycles. The CHARM2 Catalogue (Richichi et al. 2005) shows that high angular resolution measurements are available for 38 carbon stars. However, they encompass a range of subtypes and are provided with a wide spread of accuracies. To complicate matters, carbon stars experience significant changes in their diameters as a function of the pulsational phase, and it is very rarely possible to monitor their characteristics throughout their cycles. As a result of this paucity of information, each new, accurate determination is a valuable addition to the scarce observational database with which we can check theoretical estimates of carbon stars properties.

In this paper we present the first high angular resolution measurements by the technique of lunar occultations (LO) in the near infrared of the carbon star TU Gem. We also present new milliarcsecond resolution observations of a classical carbon star SS Vir (IRC 00217) by the same technique. From our angular diameter determinations for TU Gem and SS Vir, and with the complement of available fluxes, we are able to provide direct effective-temperature estimations for both stars. We also discuss the limits set by our observations on the presence of nearby companions and circumstellar matter.

\section{Observations and data analysis}

Two lunar occultations of the carbon stars SS Vir and TU Gem were observed with the fast IR photometer attached to the $1.2 \mathrm{~m}$ telescope at Gurushikhar, Mt. Abu. A single-element InSb detector operating in the broad $K$ band $(\lambda=2.2 \mu \mathrm{m}, \Delta \lambda \sim 0.4 \mu \mathrm{m})$ was used in both cases. The photometer was fitted with a diaphragm equivalent to $26^{\prime \prime}$ on the sky, and no chopping was used. The predicted circumstances of the events are given in Table 1. Details of the instrument can be found in Chandrasekhar (1999). Both occultation events were recorded well under clear sky conditions and with a signal-to-noise ratio $(S N R)$ well in excess of 100.

The data analysis was carried out by means of a code based on a non-linear least-square method (LSM). Details of the method can be found in Chandrasekhar \& Mondal (2001), as well as in Richichi et al. (1992). The time response of the $\mathrm{InSb}$ detector and preamplifier electronics to a fast gradient was determined by applying a square pulse to an IR LED and detecting the signal. This was done at approximately the same signal 
Table 1. Circumstances of the lunar occultation events.

\begin{tabular}{lll}
\hline \hline & SS Vir & TU Gem \\
\hline Occultation & Disapp. & Disapp. \\
Date (UT) & $27-04-1999$ & $08-04-2003$ \\
Event time (UT) & $18^{\mathrm{h}} 05^{\mathrm{m}} 04^{\mathrm{s}}$ & $15^{\mathrm{h}} 12^{\mathrm{m}} 00^{\mathrm{s}}$ \\
Position Angle (NESW) & $115^{\circ}$ & $118^{\circ}$ \\
Linear Rate (km s ${ }^{-1}$ ) & 0.608 & 0.636 \\
Lunar Phase (Sunlit) & $93 \%$ & $37 \%$ \\
Altitude of star & $63^{\circ}$ & $50^{\circ}$ \\
Contact Angle & $-8^{\circ}$ & $22^{\circ}$ \\
Sampling time (ms) & 1 & 1 \\
Wavelength/Bandpass $(\mu \mathrm{m})$ & $2.2 / 0.4$ & $2.2 / 0.4$ \\
Photometer Diaphragm & $26^{\prime \prime}$ & $26^{\prime \prime}$ \\
Photometric Phase & 0.55 & 0.20 \\
\hline
\end{tabular}

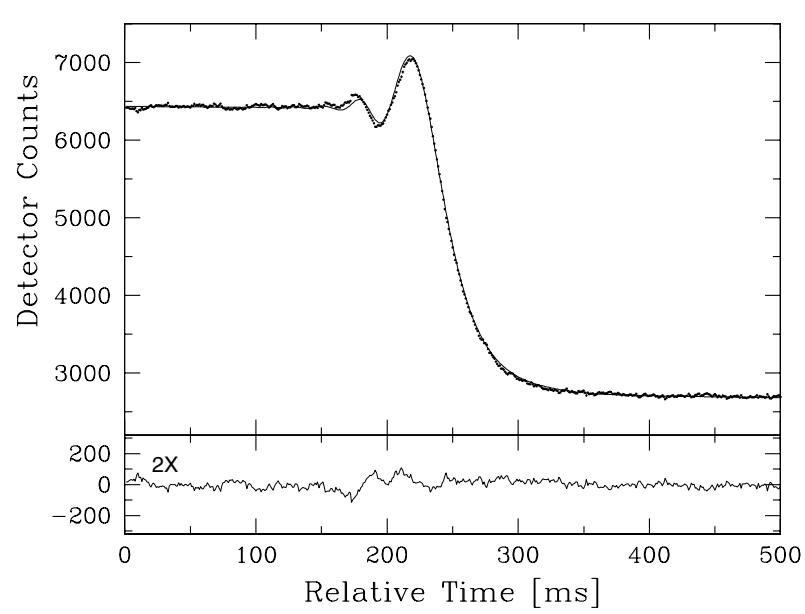

Fig. 1. Upper panel: SS Vir occultation data and fit by a LSM model (dots and solid line, respectively). Lower panel: fit residuals, enlarged by a factor of 2 for clarity.

level as the star to ensure a faithful reproduction of the time response curve that was subsequently used in the analysis.

The LSM code is well suited for cases in which a model can be provided for the source, and can be characterized by a few parameters. However, a model-independent approach is needed for cases in which it is required to investigate the presence and structure of circumstellar emission and of possible faint companions not immediately inferred from a visual inspection of the data. For this, we have used the CAL method (Richichi 1989), which iteratively applies a Lucy-Richardson deconvolution algorithm.

\section{Results and discussion}

The LO data and our best model fits for SS Vir and TU Gem are shown in Figs. 1 and 2, respectively. The resulting angular diameter results are listed in Table 2, together with some associated quantities directly related to our fits such as the ratio of observed to predicted lunar rate, the local slope of the lunar limb and the SNR of the data. The local limb slope for SS Vir could not be computed, a not uncommon circumstance when the contact angle is close to $0^{\circ}$ and the observed rate is slightly higher than predicted. The table also reports the bolometric fluxes and effective temperatures, as discussed below.

The available classifications for the cool carbon star SS Vir are listed in Table 2. Our data for SS Vir are well fitted with a single uniform-disk source with an angular diameter of

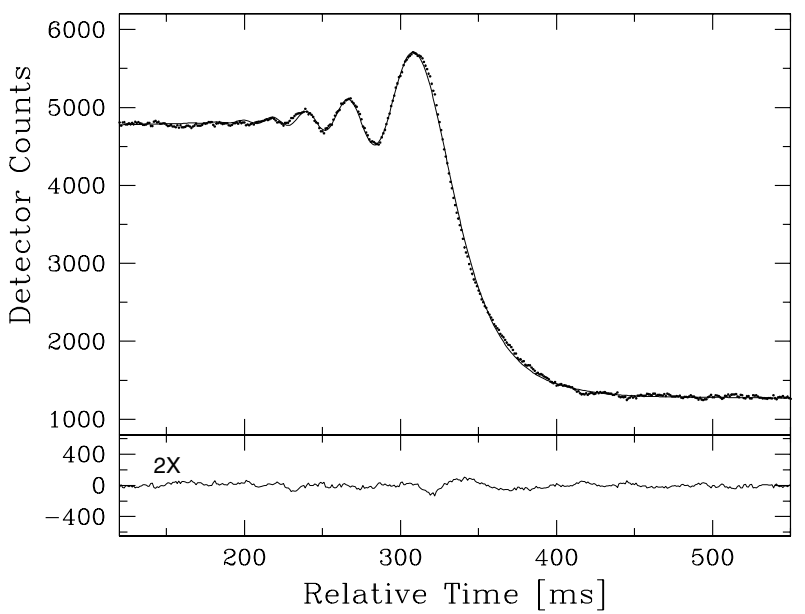

Fig. 2. Same as Fig. 1, for TU Gem. Notice the higher fringe contrast with respect to the light curve of SS Vir, indicating a smaller angular size.

Table 2. Summary of results and stellar parameters.

\begin{tabular}{lll}
\hline \hline & SS Vir & TU Gem \\
\hline Spectral type & ${\mathrm{C} 6.3^{1},{\mathrm{C} 5 \mathrm{II}^{2},},}{\mathrm{C} 6 \mathrm{II}^{2}, \mathrm{CV}^{3}}^{3}$ \\
& $\mathrm{CV6}^{3}$ & \\
Ang. Diameter (mas) & $8.37 \pm 0.07$ & $4.62 \pm 0.15$ \\
Obs./Pred. linear rate & $8.4 \%$ & $-11.0 \%$ \\
Limb slope & $-^{a}$ & $12^{\circ}$ \\
SNR & 193 & 123 \\
Bol. Flux $\left(10^{-14} \mathrm{~W} / \mathrm{cm}^{2}\right)$ & $8.34 \pm 0.42$ & $7.10 \pm 0.50$ \\
$T_{\text {eff }}(\mathrm{K})$ & $2445 \pm 41$ & $3162 \pm 107$ \\
Adopted distance $(\mathrm{pc})$ & $560^{4}$ & $515^{4}$ \\
Linear radius $\left(R_{\odot}\right)$ & 504 & 256 \\
\hline
\end{tabular}

${ }^{a}$ Cosine of observed contact angle $>1$. References: ${ }^{1}$ Bidelman (1954); ${ }^{2}$ Richer (1971); ${ }^{3}$ Bergeat et al. (2001); ${ }^{4}$ Bergeat \& Chevallier (2005).

$8.37 \pm 0.07$ milliarcseconds (mas). No circumstellar structure is detected in our analysis with the model-independent method CAL, nor is binarity. Our fit sets a limit of less $<1 \%$ of the total $K$-band flux on circumstellar matter in the range $15-180$ mas, or about 2-20 stellar diameters. Similarly, we can exclude a companion with a brightess ratio that is higher than 1:100. However, the presence of circumstellar material around SS Vir is known from various observations that addressed larger angular scales and/or different wavelength regions. The IRAS low resolution spectrum of SS Vir indicates the presence of a carbon-rich envelope. The theoretical models of Blanco et al. (1998) predict a circumstellar shell with an optical thickness of 0.3 at $5500 \AA$, inner radius of the circumstellar envelope at $3 R_{*}$ and outer radius at about $10^{4} R_{*}$. Mass loss rates of $3 \times 10^{-7} M_{\odot} / \mathrm{yr}$ are predicted, however no extended circumstellar shell has been detected in the IRAS data (Young et al. 1993). The ISO spectroscopic data (Aoki et al. 1999) show a strong emission at $11 \mu \mathrm{m}$ due to $\mathrm{SiC}$ grains and a broad absorption at $13.7 \mu \mathrm{m}$ due to $\mathrm{C}_{2} \mathrm{H}_{2}$ that originates in the photosphere or in a warm layer close to the photosphere.

It is interesting to note that observations by pupil masking (Tuthill et al. 1999) resulted in a uniform disk (UD) continuum diameter at $0.833 \mu \mathrm{m}$ of $19.5 \pm 2.0$ mas, at a photometric phase of 0.06. The authors state that this result is not of sufficiently high quality, nevertheless it clearly shows a much larger characteristic size than observed by us. This is probably due 
to the effect of short-wavelength scattering from circumstellar material, which would be consistent with polarization observations in $B$ and $V$ (Serkowski et al. 2001). On the other hand, early $H$ band LO observations (Ridgway et al. 1982) yielded a UD value of $8.71 \pm 0.49$ mas at a photometric phase close to minimum (0.56), which is in excellent agreement with our determination. It should be noted that our observations are also at nearly the same phase $(0.55$, as computed using the phase provided by Tuthill et al. 1999). We conclude that the envelope around SS Vir is not prominent in the near-IR, and we did indeed detect the actual stellar photosphere in our measurements. This is consistent with the finding that in general contamination of photospheric emission by circumstellar emission needs to be considered only longward of $2.5 \mu \mathrm{m}$ (Bergeat et al. 2001). These authors did not find substantial IR excess for either of the two stars considered in our study. We can then reliably attempt to determine the effective temperature of SS Vir. For this, we used the bolometric flux from Bergeat et al. (2001, see Table 2), with an error of 5\% inferred from other cases implicitly considered by the authors such as Z Psc. The resulting value is $T_{\text {eff }}=2445 \pm 40 \mathrm{~K}$, in good agreement with an estimate of $2400 \pm 75 \mathrm{~K}$ by A more recent estimate of $2560 \mathrm{~K}$ has been provided by Bergeat \& Chevallier (2005), although without an explicit error. We also mention that, based on theoretical modeling, these authors estimate a distance of 560 pc to SS Vir, which would imply a linear radius of $\approx 500 R_{\odot}$.

In the case of TU Gem, our LO measurement represents the first angular diameter determination. The value we present in Table 2 has been obtained by a fit with a uniform stellar disk without needing to include a circumstellar component, while considerations very similar to those made for SS Vir also apply here. In particular, although an infrared excess is known from IRAS data (Young et al. 1993; Zuckerman 1993), there seems to be little contamination of the photospheric flux in the $K$ band. It should also be noted that the classification of TU Gem is slightly less cool than that of SS Vir, for which the incidence of the circumstellar component has already been discussed and neglected earlier. We can set an upper limit to the flux of circumstellar emission of about $2 \%$ in the range 15-120 mas, or 3-25 stellar diameters.

Numerous entries exist in the literature for the magnitude of TU Gem at various wavelengths. We used the work of Taranova \& Shenavrin (2004) who provide average and variations in the JHKLM passbands over 4 pulsational periods. We then assembled an average spectral energy distribution (SED), including amplitude of variations, by adding data in the visible and from IRAS. We fitted the SED by a simple two black-body model including extinction, and we found a total bolometric flux of approximately $(7.1 \pm 0.50) \times 10^{-14} \mathrm{~W} / \mathrm{cm}^{2}$. Bergeat \& Chevallier (2005) provide estimates of the absolute bolometric magnitude and distance of $-4.66 \mathrm{mag}$ and $515 \mathrm{pc}$, respectively. This implies a flux consistent with our estimate. The resulting effective temperature is $3162 \pm 107 \mathrm{~K}$. This is somewhat higher than the value of $2715 \mathrm{~K}$ estimated by Bergeat \& Chevallier (2005), or the value of $2800-2830 \mathrm{~K}$ by Ulla et al. (1997). Changes in effective temperature are of course expected with the photometric variations, and in this respect we note that our measurement was obtained close to maximum, when the star is smaller and hotter. We also note that Ulla et al. (1997) included TU Gem in their sample of peculiar cool $\mathrm{CH}$ giants in the galactic halo, which exhibit relatively blue colors and lower metallicity and/or higher temperatures than the bulk of carbon stars. However, if TU Gem is at all a peculiar $\mathrm{CH}$ giant, it seems to be at the limit of the classification in terms of color, of mass loss (Olofsson et al. 1993; Schoier \& Olofsson 2001), and of its likely association to the galactic disc (Olofsson et al. 1993).

In order to reconcile the given flux with $T_{\text {eff }}=2800 \mathrm{~K}$, the angular diameter should be 5.9 mas, which can be excluded from our data. Alternatively, our diameter would only be consistent with $T_{\text {eff }}=2800 \mathrm{~K}$ if the flux was about $60 \%$ of the value in Table 2. In an effort to resolve this controversy, we have inspected a number of medium-resolution near-IR spectra of carbon stars published by Joyce (1998). The spectrum of TU Gem, when compared to other stars in a range of temperatures, seems more consistent with $2800 \mathrm{~K}$ than with $3100 \mathrm{~K}$, however the differences are not very compelling. We note that the spectrum published by Joyce (1998) was obtained in September 1991. The date is close to a maximum, but it also corresponds to a variability cycle that seems to have been the faintest, in terms of $V$ magnitude, of at least the last 15 years.

TU Gem is listed in the Tycho 2 Double Star Catalog (TDSC 14074, Fabricius et al. 2002). Two components A and B are mentioned, with separation 0.38 at position angle $99^{\circ} .9$ at the epoch of the observations (Hipparcos satellite, 1991-92). The same proper motion for both suggests an association. As the positions of the components are precisely known, we can compute the expected projected separation along the position angle of our LO event, which would be 329 mas, or about $1.1 \mathrm{~s}$ away from the companion (i.e. outside the section of the lightcurve shown in Fig. 2). We searched our data over a range \pm 3.5 times the expected projected separation using the integral plot method (originally introduced by P. Bartholdi, see Dunham et al. 1973), without finding convincing evidence of a companion. We also carried out a detailed analysis with the CAL method. The data could be consistent with a faint companion $(\Delta K=4.4)$ at just 17 mas separation, but we do not consider this as a positive detection since the signal causing it is at the same level of a few fluctuations in the lightcurve caused by scintillation. Instead, we adopt this value as an upper limit to the flux of any possible companion.

A possible explanation for our non-detection is that the B component was at an entirely new position at the epoch of our observation compared to the Hipparcos position, as a result of its orbital motion during $\approx 12$ years. It is not possible to draw many conclusions in this respect, since both position angle and separation might have changed. The situation is further complicated by the uncertainty in the distance to TU Gem. For example, based on the period luminosity relation, Schoier \& Olofsson (2001) estimated a significantly smaller value of $330 \mathrm{pc}$, which would imply a projected separation of just $5 \mathrm{AU}$. At the other extreme, Yuasa et al. (1999) estimated a distance of $1200 \mathrm{pc}$ using an analysis based on IRAS and radio data. This would considerably lengthen the expected binary period. In general, we expect that the range scanned in our analysis was sufficiently wide to account for significant changes in the separation, and therefore we concentrate on the hypothesis that the negative detection is due to the intrinsic color of the companion.

The TDSC magnitudes for the $\mathrm{A}$ and $\mathrm{B}$ components are $V_{\mathrm{T}}=$ 7.87 and 9.54 , and $B_{\mathrm{T}}=12.39$ and 12.74 , respectively. The Tycho passbands are sufficiently close to the Johnson passbands, and we neglect the difference hereafter. The $B-V$ colors are indicative of significant reddening, probably of a local origin given the the nature of the star, and cannot be used to speculate on the color of the companion. The $K$ magnitude of TU Gem has been monitored with repeated observations over 4 periods by Taranova \& Shenavrin (2004) who found an average value of $0.92 \mathrm{mag}$ with variations that are negligible for our purpose. Our upper limit then puts the companion at $K \geq 5.3 \mathrm{mag}$. Correspondingly, we have $V-K=6.95$ for the primary and 
$V-K \leq 4.2$ for the secondary. The former is consistent with a cool giant star, but the latter unfortunately does not constrain the spectral type of the companion sufficiently. Therefore, we can neither prove the presence of a white dwarf companion as expected from more typical $\mathrm{CH}$ stars nor deny it.

\section{Conclusions}

We have presented new, accurate measurements by lunar occultations of the two cool carbon stars SS Vir and TU Gem. For the former, we derive an angular diameter of $8.37 \pm 0.07$ mas, corresponding to about 500 solar diameters. We also derived an effective temperature of $2445 \mathrm{~K}$, in general agreement with indirect and model-dependent estimates. We put quantitative upper limits on the circumstellar flux and on the presence of companions.

For TU Gem we convincingly determine an angular diameter of $4.62 \pm 0.15$ mas. Using available flux estimates, this leads to a temperature of $3160 \pm 110 \mathrm{~K}$, which seems difficult to reconcile with estimates of $2715-2830 \mathrm{~K}$ from the literature. Also, we cannot detect the companion listed in the Tycho 2 Double Star Catalog directly. We place an upper limit of $\Delta K \geq 5.3$ on the brightness of the companion and we discuss its color, without however being able to significantly constraint its spectral type and the hypothesis of a much bluer star than the primary.
Acknowledgements. This research has made use of the Simbad database, operated at the Centre de Donnés Astronomiques de Strasbourg (CDS), and of NASA's Astrophysics Data System Bibliographic Services (ADS). Part of the work was carried out during a stay of TC in Garching, supported by the ESO Visitor Program.

\section{References}

Aoki, W., Tsuji, T., \& Ohnaka, K. 1999, A\&A, 350, 945 Bergeat, J., Knapik, A., \& Rutily, B. 2001, A\&A, 369, 178 Bergeat, J., \& Chevallier, L. 2005, A\&A, 429, 235 Bidelman, W. P. 1954, ApJSS, 1, 175

Blanco, A., Borghesi, A., Fonti, S., \& Orofino, V. 1998, A\&A, 330, 505 Chandrasekhar, T. 1999, BASI, 27, 43

Chandrasekhar, T., \& Mondal, S. 2001, MNRAS, 322, 356

Dunham, D. W., Evans, D. S., McGraw, J. T., Sandmann, W. H., \& Wells, D. C. 1973, AJ, 78,482

Fabricius, C., Høg, E., Makarov, V. V., et al. 2002, A\&A, 384, 180

Joyce, R. R. 1998, AJ, 105, 2059

Olofsson, H., Eriksson, K., Gustafsson, B., \& Carlstrom, U. 1993, ApJS, 87, 267

Richer, H. B. 1971, ApJ, 167, 521

Richichi, A. 1989, A\&A, 226, 366

Richichi, A., Di Giacomo, A., Lisi, F., \& Calamai, G. 1992, A\&A, 265, 535

Richichi, A., Percheron, I., \& Khristoforova, M. 2005, A\&A, 431, 773

Ridgway, S. T., Jacoby, G. H., Joyce, R. R., Siegel, M. J., \& Wells, D. C. 1982, AJ, 87, 808 Schoier, F. L., \& Olofsson, H. 2001, A\&A, 368, 969

Serkowski, K., \& Shawl, S. J. 2001, AJ, 122, 2017

Taranova, O. G., \& Shenavrin, V. I. 2004, Astr. Lett., 30, 549

Tuthill, P. G., Haniff, C. A., \& Baldwin, J. E. 1999, MNRAS, 306, 353

Ulla, A., Thejll, P., Kipper, T., \& Jorgensen, U. G. 1997, A\&A, 319, 244

Young, K., Phillips, T. G., \& Knapp, G. R. 1993, ApJS, 86, 517

Yuasa, M., Unno, W., \& Magono, S. 1999, PASJ, 51, 197

Zuckerman, B. 1993, A\&A, 276, 367 